\title{
Vortex Dynamics around Pitching Plates
}

Ryan T. Jantzen, ${ }^{1, a)}$ Kunihiko Taira, ${ }^{1, b)}$ Kenneth O. Granlund, ${ }^{2, c)}$ and Michael V. $\mathrm{Ol}^{2, \mathrm{~d})}$

1) Department of Mechanical Engineering and Florida Center for Advanced Aero-Propulsion, Florida State University, Tallahassee, Florida 32310, $U S A$

${ }^{2)}$ Aerospace Systems Directorate, U.S. Air Force Research Laboratory, Wright-Patterson Air Force Base, Ohio 45433, USA

(Dated: 29 April 2014)

Vortex dynamics of wakes generated by rectangular aspect-ratio 2 and 4 and twodimensional pitching flat plates in free stream are examined with direct numerical simulation and water tunnel experiments. Evolution of wake vortices comprised of tip, leading-edge and trailing-edge vortices is compared with force history for a range of pitch rates. The plate pivots about its leading edge with reduced frequency from $\pi / 8$ to $\pi / 48$, which corresponds to pitching over 1 to 6 chord lengths of travel. Computations have reasonable agreement with experiments, despite large differences in Reynolds number. Computations show that the tip effects are confined initially near the wing tips, but begin to strongly affect the leading-edge vortex as the motion of the plate proceeds, with concomitant effects on lift and drag history. Scaling relations based on reduced frequency are shown to collapse aerodynamic force history for the various pitch rates.

PACS numbers: Vortex dynamics (fluid flow), 47.32.C-, Separated flows, 47.32.Ef-, Wakes, laminar, 47.15.Tr-

\footnotetext{
a)Electronic mail: rtj09@my.fsu.edu

b) Electronic mail: ktaira@fsu.edu

c)Electronic mail: kenneth.granlund.1.ctr@us.af.mil

d)Electronic mail: michael.ol@us.af.mil
} 


\section{INTRODUCTION}

The humble rigid flat plate remains a useful abstraction for the study of separated flow with unsteady boundary conditions, for applications of aircraft undergoing agile maneuvers and operating in harsh environments such as gusts. Various types of small to large unmanned air vehicles with fixed, rotating, or flapping wings ${ }^{1,2}$ oftentimes take inspiration from biological flyers to take advantage of vortical effects for enhanced lift. In gusty environments, the effective free stream velocity and the angle of attack can change within a short period of time ${ }^{3}$, which with a buoyancy correction is the inverse problem to that of maneuver in steady freestream ${ }^{4}$.

Flow separation and the resulting vortex formation are inherently nonlinear phenomena, with nontrivial deviations from classical unsteady theories ${ }^{5,6}$ that motivate an aim for detailed understanding of the physics to predict the flow field and the aerodynamic forces ${ }^{7}$. Study of the vortex dynamics around plates undergoing unsteady motions has been identified as an international research task by the NATO AVT-202 Group and the AIAA Fluid Dynamics Technical Committee's Low Reynolds Number Discussion Group. The identified research task encompasses a wide variety of motions, including pitching ${ }^{8,9}$, $\operatorname{rotation}^{10,11}$, various acceleration profiles ${ }^{12,13}$, and the combination thereof ${ }^{14}$.

There have been extensive experimental studies on the pivot point location for purely pitching plates. Two-dimensional flat plates were studied using direct force measurements and qualitative dye flow visualization by Granlund et al. ${ }^{15}$ to compare the flow field evolution and aerodynamic forces for pitching maneuvers over a range of reduced frequencies and pivot point locations. They were able to correlate lift and drag coefficients as functions of both angle of attack and pivot point location for reduced frequencies greater than 0.1. For finite-aspect ratio wings, $\mathrm{Yu}$ and Bernal ${ }^{16}$ studied the effect of pivot point location and reduced frequency on the flow structure and aerodynamic forces for an $A R=4$ pitching flat plate using direct force measurements and two-dimensional PIV velocity measurements. Granlund et al. ${ }^{17}$ performed an extensive parametric study on the influence of aspect ratio, pivot-point location, and reduced frequency for rotational and translational accelerating flat plates from $R e=14$ to 10,000. For all Reynolds numbers considered, both non-circulatory and circulatory loading resulting from the acceleration and deceleration of the plate were found to be highly dependent on the pivot-point location; for instance, a more forward pivot point produces a higher peak lift due to an induced camber effect ${ }^{5}$.

On the numerical side, Taira and Colonius ${ }^{7}$ used direct numerical simulations to analyze the three-dimensional separated flow over low-aspect-ratio flat plates in translation. They observed that the tip vortices from the low-aspect-ratio flat plates help stabilize the separated flow for impulsively started plates. The stability of the wake dynamics was also characterized over a wide range of aspect ratios and angles of attack. High-fidelity simulations were performed to investigate the effect of reduced frequency and Reynolds number on the flow structure and unsteady loading for pitching flat plates of $A R=2$ and spanwise periodic configuration by Visbal ${ }^{18}$ and Garmann and Visbal ${ }^{19}$, respectively. They found that for all pitch rates considered, there is a significant increase in the maximum lift achieved compared to plates at static angles of attack. Visbal ${ }^{18}$ noted that the increase in lift for finite-aspectratio plates can be attributed to the three-dimensional dynamic stall process resulting from the formation of a LEV that evolves into an arch-type vortex. He showed the flow fields to be qualitative similar over $R e=1,000$ to 40,000 . In a companion study to Granlund et al. ${ }^{17}$, Jantzen et al. ${ }^{20}$ reported on the three-dimensional wake dynamics and unsteady forces for 
both pitching and accelerating low-aspect-ratio plates. It was found that reduced tip effects for higher-aspect-ratio plates influence the formation and evolution of the LEV.

The current investigation examines the vortex dynamics around plates with aspect ratio 2 and 4 (and 2D) pitching about the leading edge, using both numerical (immersed boundary method) and experimental (water tunnel) approaches. We consider such canonical pitch-up motion that can potentially be encountered by miniature aircraft in gusty conditions to provide a better understanding of how the flow evolves around the wing and how unsteady aerodynamic forces are generated. The pitch rates are selected to bracket the range of time scales identified with the vortex formation time ${ }^{21,22}$. The present work particularly highlights the finite-aspect-ratio effects (tip effects) for pitching wings which has not been examined in detail with computations. By performing parameter studies with varied pitching frequency and plate aspect ratio, we aim to provide insights towards designing new vehicles that are able to withstand the evolving three-dimensional flow structure on the maneuvering wings and also utilize the unsteady forces generated during these motions efficiently for improved performance and stability.

The present paper is organized in the following manner. In Section II, we present the problem setup and methodology. Section III discusses the formulation and dynamics of the vortices around the pitching plate at various pitch rates and aspect ratios, to elucidate the influence of the leading-edge and tip vortices. Section IV analyzes the generation and growth of the leading-edge vortices during the pitching motion. We provide discussions on the unsteady aerodynamic forces experienced by the wing in Section V. A non-dimensional scaling that incorporates pitch rates is provided to collapse the force history during pitching. The numerical and experimental results are compared in Section VI to illustrate the differences due to Reynolds number effects. Despite the large difference in Reynolds numbers, the flow fields and force histories are found to be in qualitative agreement. Concluding remarks are offered in Section VII to summarize the findings from this study.

\section{PROBLEM SETUP}

The present investigation considers flat-plate wings with rectangular planform of various aspect ratios undergoing a pitching maneuver about the leading edge in a constant freestream. The spatial coordinates are defined with $x, y$, and $z$ representing the streamwise, vertical, and spanwise directions, respectively. Length scales are non-dimensionalized by the chord length $c$, and the velocity vector is non-dimensionalized by the freestream velocity value $U_{\infty}$. Temporal variable $t$ is the non-dimensional convective time, normalized by the freestream velocity and the chord length. The Reynolds number is $R e=U_{\infty} c / \nu$, where $\nu$ is the kinematic viscosity. The forces on the flat plate $\left(F_{x}, F_{y}\right)$ are reported as non-dimensional lift and drag, defined by $C_{L}=F_{y} /\left(\frac{1}{2} \rho U_{\infty}^{2} A\right)$ and $C_{D}=F_{x} /\left(\frac{1}{2} \rho U_{\infty}^{2} A\right)$, respectively, where $A$ is the planform area of the plate and $\rho$ is the density of the fluid.

The flat plate pitches from zero-incidence to a post-stall angle of attack $\alpha_{\max }$ in a smoothed linear ramp. Smoothing of the start and end of the pitch ramp is through a form of relationship proposed by Eldredge ${ }^{23}$ :

$$
\alpha(t)=\frac{\Omega_{\circ}}{2 a} \log \left\{\frac{\cosh \left[a\left(t-t_{1}\right)\right]}{\cosh \left[a\left(t-t_{2}\right)\right]}\right\}+\frac{\alpha_{\max }}{2},
$$

where $\Omega_{0}$ is the nominal pitch rate given by $\Omega_{0}=\alpha_{\max } / t_{p}$, where $\alpha_{\max }$ is the maximum angle 
of attack at the end of the pitching motion (taken in all cases as $45 \mathrm{deg}$ ), and $t_{p}=t_{2}-t_{1}$ is the pitching interval. Here, $t_{1}=0$ and $t_{2}$ is determined by the reduced frequency $(K \equiv$ $\left.\Omega_{0} c / 2 U_{\infty}=\alpha_{\max } c / 2 U_{\infty} t_{p}\right)$ of the pitching motion, $t_{2}=t_{1}+\alpha_{\max } c /\left(2 U_{\infty} K\right)$. The parameter $a$ is a smoothing parameter chosen to regularize the sharp jump in $\ddot{\alpha}$. The time history of the pitch incidence angle $\alpha$ is illustrated in Figure 1 for different rates. This motion profile has been selected to be the test case for a collective study by the NATO AVT-202 working group. Throughout this paper, we refer to the different pitch-rate cases as $C N$, where $N$ indicates the convective time units (chord lengths) over which the plate pitches.

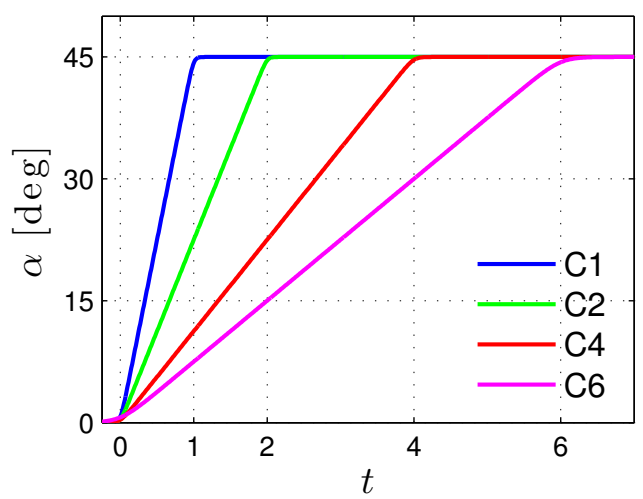

FIG. 1: The smoothed linear ramp used for the pitching maneuver from $\alpha=0^{\circ}$ to $45^{\circ}$.

The smoothing parameter is chosen to be $a=21,16,11$, and 4 , for the cases of $C 1, C 2$, $C 4$, and $C 6$, respectively. These values are selected to match work by Visbal ${ }^{18}$ and Yilmaz and Rockwell ${ }^{24}$. In numerical simulations, we consider rectangular wings of aspect ratios 2 and 4 and the two-dimensional case for the freestream Reynolds number $R e=300$, which is a value chosen to elude issues of turbulence and to highlight the large-scale wake structures generated by the unsteady motion of the wing. The reduced frequency $K=\pi / 8, \pi / 16$, $\pi / 32$, and $\pi / 48$ correspond to the cases of $C 1, C 2, C 4$, and $C 6$, respectively.

\section{A. Computational setup}

Three-dimensional incompressible flow over the flat-plate wing is numerically solved with the immersed boundary projection method ${ }^{25}$, which creates a plate in the domain with a set of Lagrangian points where appropriate boundary forces are introduced to enforce the no-slip condition on the wing surface. This method has been used to simulate a wide variety of flows and has been well-validated ${ }^{7,25,26}$. We use a computational domain with a typical size of $(x, y, z) \in[-4,6] \times[-5,5] \times[-5,5]$. The plate is positioned in the computational domain with its midspan point on the leading edge at the origin, as shown in Figure 2 . The inlet and side boundary conditions are set to constant uniform flow with $U_{\infty}=1$ and the outlet boundary condition uses a convective boundary condition of $\partial \boldsymbol{u} / \partial t+U_{\infty} \partial \boldsymbol{u} / \partial x=0$ to allow the wake vortices to freely exit the computational domain without disturbing the near-field solution. A simulation is first performed to determine the steady-state flow over the plate at zero degree angle of attack. This steady state is then used as the initial condition for all simulations in which the wing undergoes the pitching motion prescribed by Eq. (1). 
The thickness of the plate in the simulation is modeled to be infinitely thin with a discrete delta function.
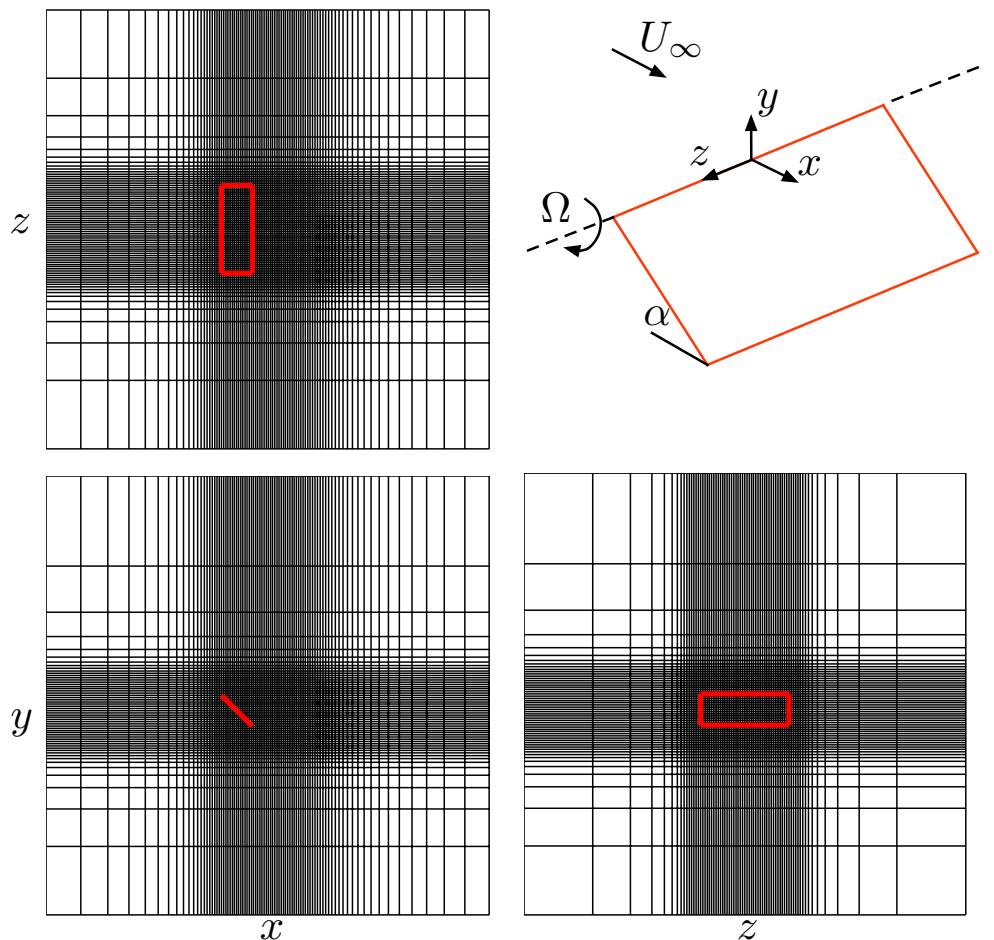

FIG. 2: The computational setup showing the $x z, x y$, and $z y$ grid planes with every fourth gridline and the $A R=2$ pitching plate shown.

To ensure numerical simulations are performed with sufficient spatial grid resolution, we perform a pitching plate study but with varying grid sizes. Shown in Figure 3 gives the lift and drag histories with varied grid resolutions from a case where the $A R=2$ plate is pitching over one chord length of travel $(C 1)$ for $R e=300$ with grid sizes listed in Table I. The results in Figure 3 and Table I show that the medium size grid provides sufficient resolution to achieve convergence. This is also illustrated by the flowfield images shown on the right side of Figure 3. For the three cases, we essentially see no observable differences in the vortical structures. For all cases discussed below, the resolution used in this study is based on the medium resolution. The computational domain size is increased with added points in the spanwise direction for $A R=4$ cases. The CFL number is limited to 0.5 in all cases.

\begin{tabular}{cccc}
\hline Resolution & Coarse & Medium & Fine \\
\hline \hline$n_{x} \times n_{y} \times n_{z}$ & $141 \times 91 \times 116$ & $170 \times 110 \times 140$ & $212 \times 137 \times 175$ \\
$\frac{\max \left|C_{L}-C_{L, \text { fine }}\right|}{\max \left|C_{L, \text { fine }}\right|}$ & $6.9 \%$ & $3.0 \%$ & - \\
$\frac{\max \left|C_{D}-C_{D, \text { fine }}\right|}{\max \left|C_{D, \text { fine }}\right|}$ & $11.2 \%$ & $5.7 \%$ & - \\
\hline
\end{tabular}

TABLE I: Number of grid points used for the grid resolution study. 

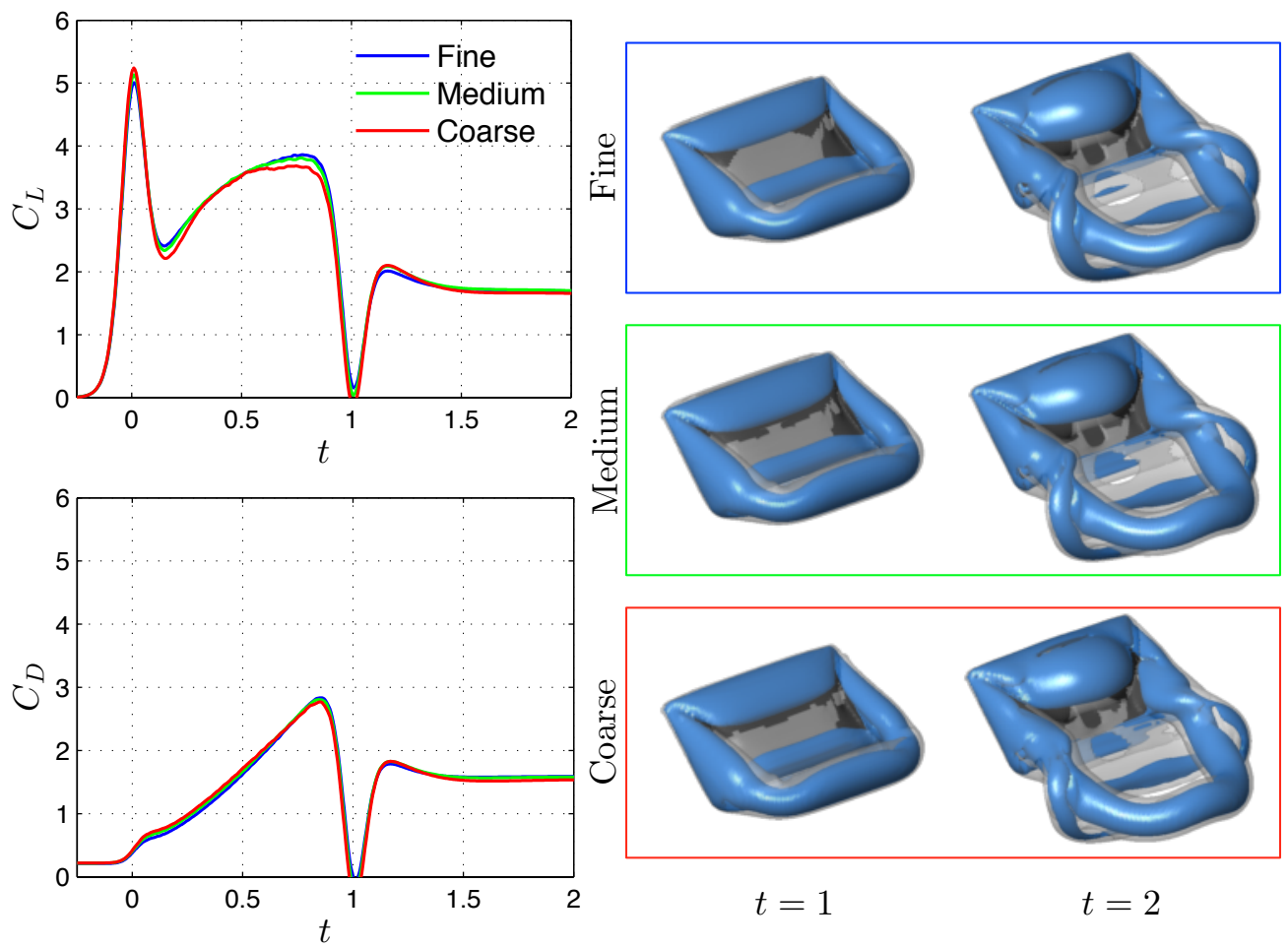

FIG. 3: Grid resolution study showing the lift (top left) and drag (bottom left) force coefficients along with snapshots of the instantaneous $Q$-criterion $(Q=3)$ and vorticity magnitude $(\|\boldsymbol{\omega}\|=3)$ showing the grid dependence on the three-dimensional flow structure for the $A R=2$ flat plate.

\section{B. Experimental Setup}

Direct force measurement and fluorescent dye flow visualization for pitching plates at $R e=20,000$ were conducted in the Horizontal Free-surface Water Tunnel at the U.S. Air Force Research Laboratory at Wright-Patterson Air Force Base ${ }^{27}$. The tunnel has 4:1 contraction ratio and $0.46 \mathrm{~m}$ wide by $0.61 \mathrm{~m}$ high test section, speed range of 3 to $45 \mathrm{~cm} / \mathrm{s}$, and $u$-component turbulence intensity of $0.4 \%$ at $15 \mathrm{~cm} / \mathrm{s}$. The tunnel is fitted with a threedegree of freedom electric rig, consisting of a triplet of $\mathrm{H} 2 \mathrm{~W}$ linear motors, driven by AMC DigiFlex servo-drives controlled by a Galil DMC 4040 4-channel card, with user-selected proportional/integral/derivative (PID) constants for each channel. The model motion of pitch and plunge are controlled via two motors mounted vertically on a plate above the tunnel test section, shown in Figure 4 (left). For the present study, $A R=2$ and 4 flat plates are used with a chord length of $117 \mathrm{~mm}$ and $75 \mathrm{~mm}$, respectively. The thickness of the plate is $1.59 \mathrm{~mm}(1 / 16 \mathrm{in})$.

Flow visualization is limited to qualitative inferences from dye illuminated by planar laser fluorescence. Rhodamine $6 \mathrm{G}$ dissolved in water is injected at the leading and trailingedge $3 / 4$ semispan location by a positive-displacement pump, connecting to a set of $0.5 \mathrm{~mm}$ internal-diameter rigid lines glued to the surface of the plate, in an approach similar to that of Ol et al. ${ }^{27}$ The dye is illuminated by an Nd:YLF $527 \mathrm{~nm}$ pulsed laser sheet of $\approx 2 \mathrm{~mm}$ thickness at $50 \mathrm{~Hz}$, and images are recorded with a PCO DiMax high-speed camera through 

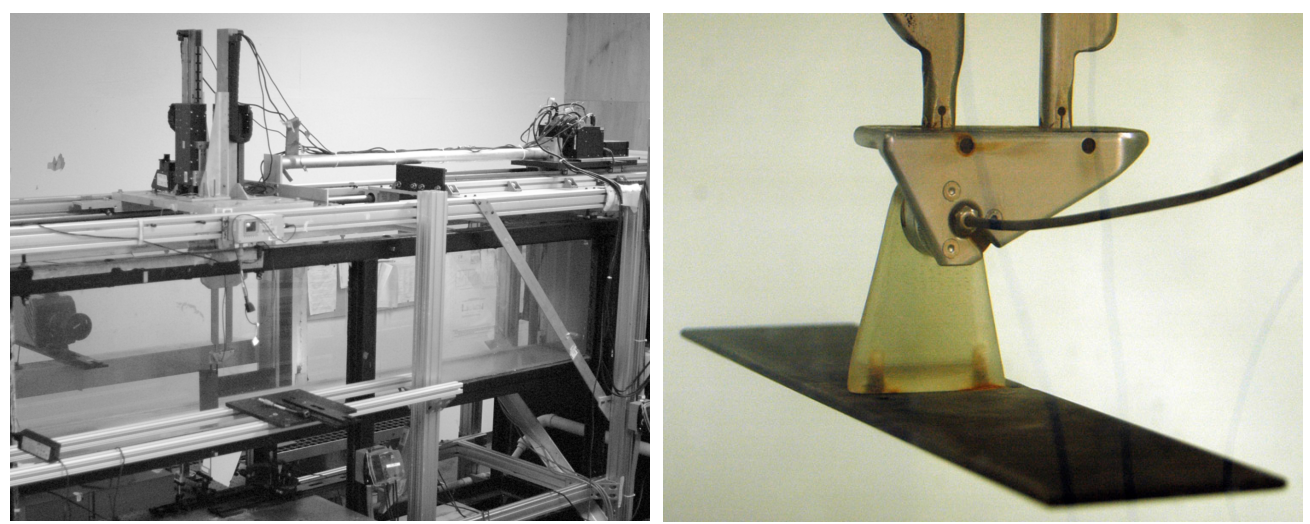

FIG. 4: The experimental setup in the U.S. Air Force Research Laboratory Water Tunnel. Water tunnel with motion mechanism (left) and flat-plate model (right).

a Nikon PC-E 45mm Micro lens. A Tiffen orange \#21 filter is used to remove the incident and reflected laser light, leaving only the fluorescence.

Force data are recorded from an ATI Nano-17 IP68 6-component integral load cell. The load cell is visible in Figure 4 (right) at the junction of the triangular mounts. Load cell strain gage electrical signals are A/D converted in an ATI NetBox interface and recorded using a Java application, and are filtered in three steps. The first is a low-pass filter in the ATI NetBox at $f=34 \mathrm{~Hz}$, to avoid introducing noise not correlated with motion force data as well as structural eigenfrequency. These are on the order of $50 \mathrm{~Hz}$. The second step uses a moving-average of 11 points to smooth the data and to numerically stabilize the third filtering operation, which is the fourth order Chebychev II low-pass filter with - 20dB attenuation of the stopband. The cutoff frequency is five times the motion frequency, were the ramp motion to have been a 1/4-sine wave. In the present study, the force measurements have an uncertainty level of $<2 \%$ of the steady state values (of those after the pitch-up motion ends). The uncertainty in experimental measurements were obtained from loadcell quoted uncertainty and the temporal average of 10 repetitions of each experimental case.

\section{VORTEX DYNAMICS}

Here, we focus on the evolution of the vortical structures around the wing and the effects that the pitch rate and aspect ratio have on the vortex dynamics. The three-dimensional vortical structures for the $A R=2$ and 4 flat plates are shown in Figure 5 for the pitching case occurring over one chord of travel $(C 1)$, visualized by the iso-surface of the $Q$-criterion $(Q=3)$ in blue and the iso-surface of the magnitude of vorticity $\left(\|\boldsymbol{\omega}\|_{2}=3\right)$ in gray. The $Q$-criterion shows the vortex cores and the vorticity norm highlights the vortex sheets. The values for the iso-surfaces were chosen based on previous studies of low Reynolds number simulations ${ }^{7}$.

As the plate begins to pitch and the incidence angle increases to $\alpha=11^{\circ}$, the vortex sheet over its top surface begins to roll up, initiating the formation of the LEV. As the angle further increases from $\alpha=23^{\circ}$ to $38^{\circ}$, tip vortices arise from the pressure difference between the top and bottom surfaces, creating a vortex loop with the starting vortex that has detached from the trailing edge of the plate, the two tip vortices and the LEV. The 


\begin{tabular}{c|ccccc}
$K=\pi / 8$ & $\begin{array}{c}t=0 \\
(C 1)\end{array}$ & $\begin{array}{c}t=0.25 \\
\left(\alpha=0^{\circ}\right)\end{array}$ & $\begin{array}{c}t=0.5 \\
\left(\alpha=11^{\circ}\right)\end{array}$ & $\begin{array}{c}t=0.75 \\
\left(\alpha=38^{\circ}\right)\end{array}$ & $\begin{array}{c}t=1 \\
\left(\alpha=45^{\circ}\right)\end{array}$ \\
\hline$A R=4$ & & &
\end{tabular}

FIG. 5: Instantaneous $Q$-criterion iso-surfaces $(Q=3$, blue) and magnitude of vorticity $(\|\boldsymbol{\omega}\|=3$, gray) showing the three-dimensional flow structure for the $A R=4$ (top) and 2 (bottom) plates for the $C 1$ case at $R e=300$. The flow field is visualized for a reference frame fixed with the plate.

growth of the LEV is uniform across most the span of the plate, while the legs of the LEV stay pinned to the corners at the leading edge. By the time that the plate has reached the maximum angle of $\alpha=45^{\circ}$, the trailing-edge (starting) vortex has advected downstream. During the pitching motion, the tip vortices remain pinned to the leading-edge corners of the plate. The LEV has continued to grow, remaining fairly uniform while still being attached to the plate. In the present discussion, the LEV is considered detached ${ }^{18}$ when the legs of the LEV are no longer attached to the corners of the leading edge. We note that the definition of the detached LEV does not depend significantly on the values of the iso-surfaces chosen to visualize them. Throughout the entire motion for this pitch rate, the flow field evolution is very similar between $A R=2$ and 4 at each respective snapshot of time. As will be seen subsequently, aspect-ratio independence of the flow field is attenuated with decreasing pitch rate.

The evolution of the flow structure for all four of the pitch rates considered is given in Figures 6 and 7 for the $A R=2$ and 4 plates, respectively. To extend upon the discussion about the fastest pitching rate, $C 1$, we consider the flow structure later in time after the plate has completed its pitching maneuver $(t>1)$. By $t=2$, the tip vortices separate from the trailing edge of the plate while staying attached to the corners of the leading edge. During this process, a second vortex loop, created by the quick angular deceleration of the plate, sheds from the trailing edge which wraps around the initially generated tip vortices. By $t=3$, the LEV begins to lift off at the centerline of the plate and resembles an arch-type vortex that has been previously observed numerically by Visbal ${ }^{18}$ and experimentally by Yilmaz and Rockwell ${ }^{24}$ for $A R=2$ pitching plates at moderate Reynolds numbers.

After $t=3$, we start to observe differences in the flow structure between the $A R=2$ and 4 plates, especially in the evolution of the LEV. For the $A R=2$ plate, the LEV remains relatively close to the surface of the plate long after $(t>8)$ it has detached from the corners of the leading edge. This delayed advection is due to the presence of the stronger influence from the tip vortices on the mid-span region for the $A R=2$ plate, similar to what is observed by Taira and Colonius ${ }^{7}$. At $t=5$, we begin to notice that the tip vortices begin to roll in towards the midspan and by $t=8$, the counter rotating tip vortices have become very close to one another, creating a significant downwash which essentially pulls the LEV towards the surface of the plate, slowing down the advection of the LEV. 


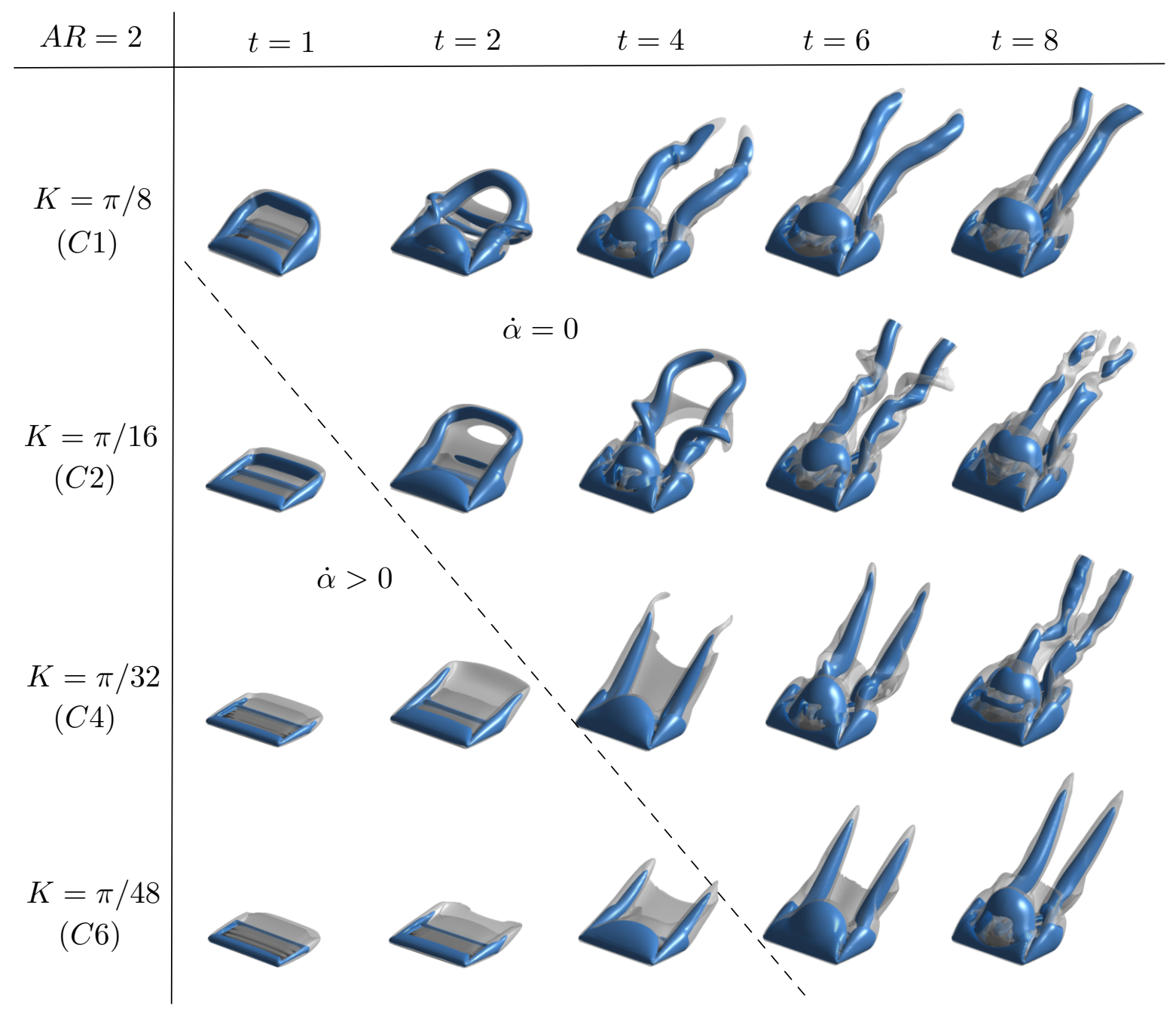

FIG. 6: Instantaneous $Q$-criterion iso-surfaces $(Q=3$, blue) and vorticity magnitude iso-surfaces $(\|\boldsymbol{\omega}\|=3$, gray) showing the three-dimensional flow structure for the $A R=2$ flat plate at various times during the four different pitching motions at $R e=300$. The flow field is visualized for a reference frame fixed with the wing. The images to the left of the dotted line correspond to when the plate is still in motion $(\dot{\alpha}>0)$, and the images to the right correspond to when the plate has reached the maximum $\alpha$ and is no longer moving

$$
\left(\dot{\alpha}=0, \alpha=45^{\circ}\right) \text {. }
$$

In contrast, for the $A R=4$ plate, the LEV lifts away from the suction-side surface and detaches from the corners at the leading edge. By $t=8$, the LEV develops into a large horseshoe vortex as it travels away from the plate due to the vortex being stretched along the streamwise and plate normal directions. Another difference that is present in the $A R=4$ case that is not observed for the $A R=2$ plate are the smaller structures that appear to wrap around the head of the horseshoe type vortex at later times $(t>5)$. These structures result from the continued roll up of the trailing-edge vortex and the large spanwise variation of the flow structure. The trailing-edge vortex (TEV) rolls up faster along the midspan compared to the $A R=2$ case, while at the tips of the plate there is a delay in this roll up due to the downwash induced by the tip vortices and the legs of the LEV on the surface of the plate. 


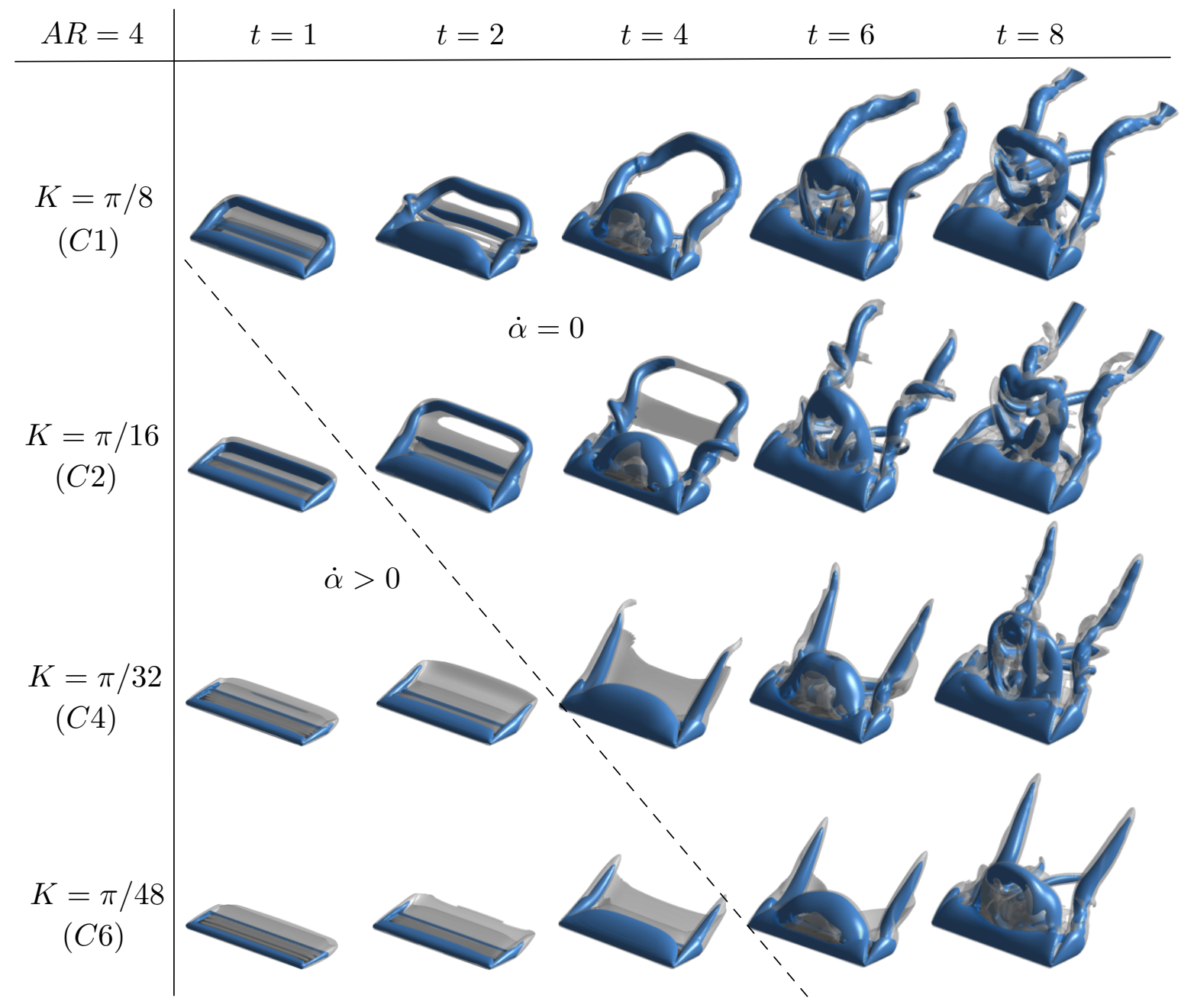

FIG. 7: Instantaneous $Q$-criterion iso-surfaces $(Q=3$, blue) and vorticity magnitude iso-surfaces $(\|\boldsymbol{\omega}\|=3$, gray) showing the three-dimensional flow structure for the $A R=4$ flat plate at various times during the four different pitching motions at $R e=300$. The flow field is visualized for a reference frame fixed with the plate. The images to the left of the dotted line correspond to when the plate is still in motion $(\dot{\alpha}>0)$, and the images to the right correspond to when the plate has reached the maximum $\alpha$ and is no longer moving

$$
\left(\dot{\alpha}=0, \alpha=45^{\circ}\right) \text {. }
$$

We next consider the influence of various pitch rates $(C 1, C 2, C 4$, and $C 6)$ for the $A R=2$ and 4 plates as shown in Figures 6 and 7, respectively. The times necessary for LEV formation and detachment are seen to depend strongly on pitch rate. For the $C 1$ case, we previously observed that the pinch-off of the LEV occurs around $t=4$, which is well after the pitching motion has stopped. It can be seen that for the $C 2$ case, the formation of the LEV also occurs well after the end of the pitching motion and it detaches between $t=4$ and 5. For the two slowest pitching rates, $C 4$ and $C 6$, the LEV forms by the end of the motion ( $t=4$ and 6 , respectively) and detaches roughly around one convective time unit afterwards for the $A R=2$ plate. In the case of $A R=4$ plate, this detachment occurs around one convective time unit after the end of the pitching motion. For the $A R=2$ 
$A R=2$
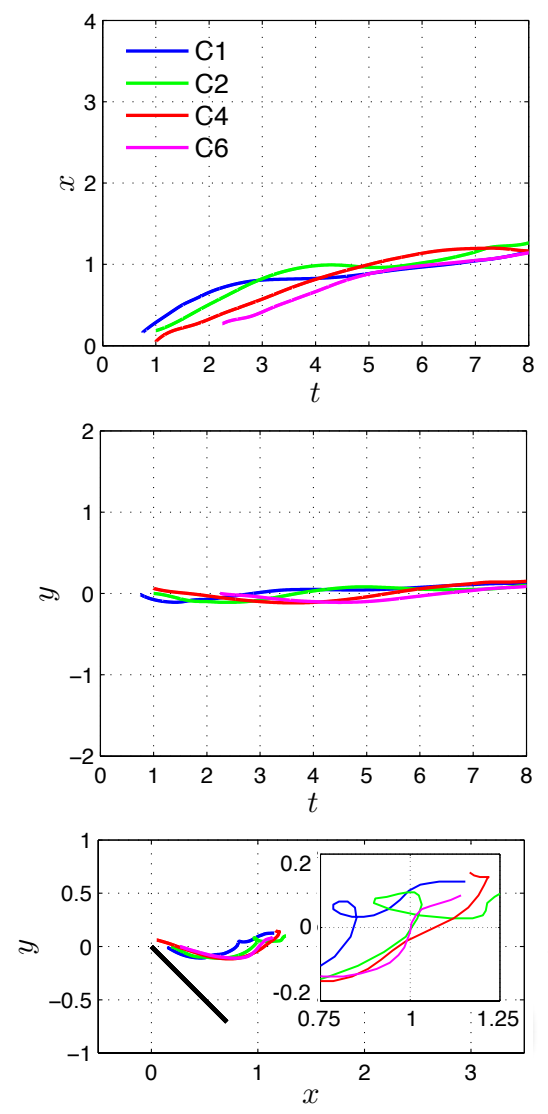

$A R=4$
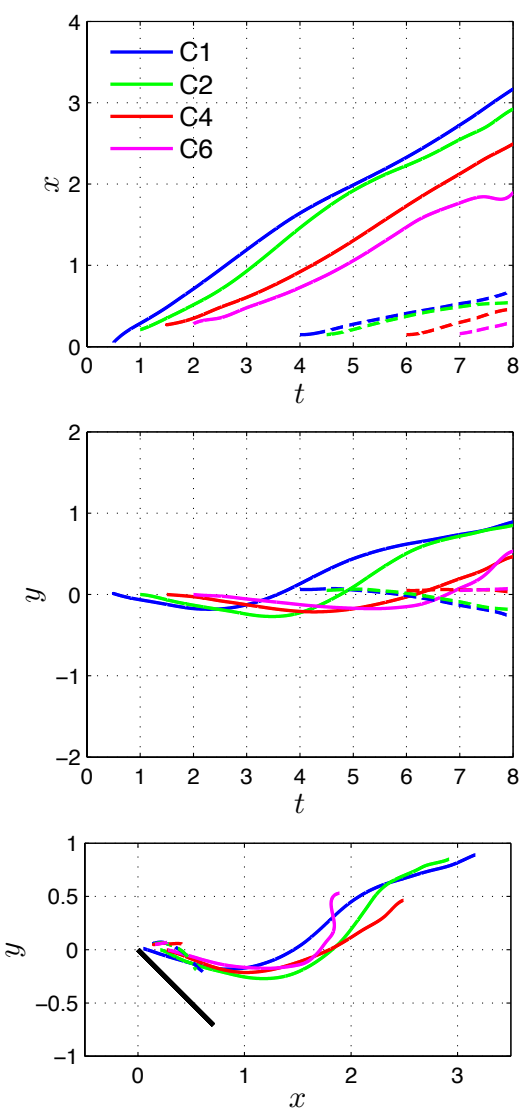

$2 \mathrm{D}$
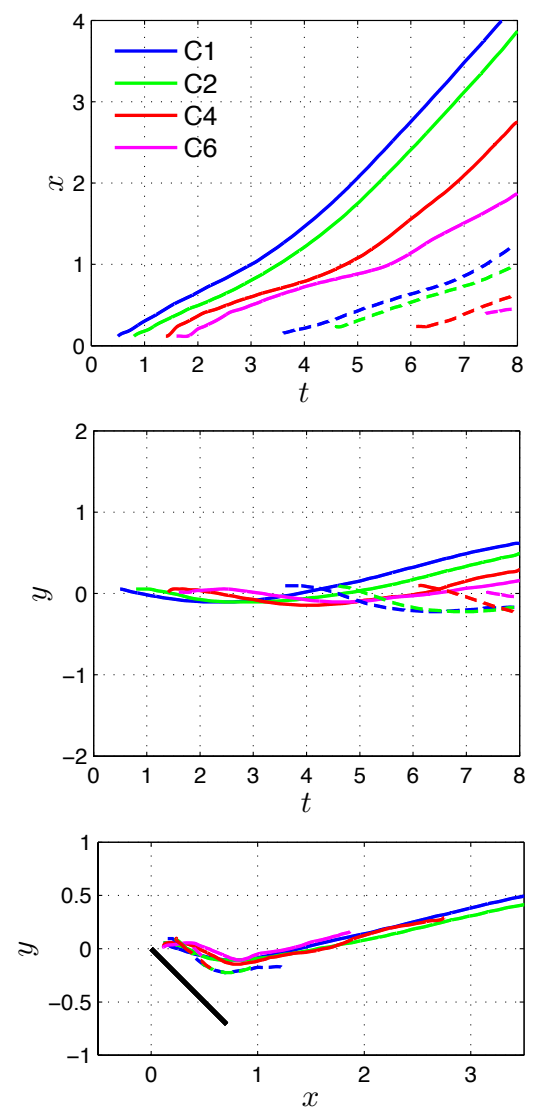

FIG. 8: Comparison of the temporal evolution of the spatial location of the LEV for the four pitch rates in the $x$-direction (top row) and $y$-direction (middle row) and the spatial location of the LEV with respect to the plate (bottom row) for the $A R=2$ (left column), 4 (center column) and 2D (right column) flat plates. The solid line represents the first LEV and the dashed line represents the formation of a second LEV. For the time period considered in this study, no additional LEVs are observed for the $A R=2$ cases.

plate, at the furthest convective time considered in the present study, the LEV is located at roughly the same location for all pitch rates considered. This is not the case for the $A R=4$ wing, since the tip vortices have a much weaker influence on the detachment of the LEV.

\section{LEADING-EDGE VORTEX TRACKING}

In order to better understand the vortex dynamics associated with the present pitching wing simulations, the vortex identification method of Graftieaux et al. ${ }^{28}$ is employed to track the formation and evolution of the LEV. The vortex identification function $\gamma_{1}$ captures the center of a vortex when its value takes the maximum value (theoretically $\max \left(\gamma_{1}\right)=1$ ). In the current study, $\gamma_{1}$ is calculated on the midspan plane. We select a threshold of $\gamma_{1} \geq 0.9$ to identify a vortex and track its center where the maximum spatial value of $\gamma_{1}$ is attained.

The trajectory of the LEV centroid on the midspan plane for all pitch rates considered for the $A R=2,4$, and 2D plates are given in Figure 8. We note that the calculation of the LEV centroid begins at different times for the four different pitch rates, due to the time it 
takes for the LEV to initially form (with $\gamma_{1} \geq 0.9$ ). For the $A R=2$ plate, the LEV centroid travels from the leading edge in roughly the same direction for all four pitch rates, until it advects one chord length downstream. For the two fastest pitch rates, $C 1$ and $C 2$, the LEV actually moves upstream before continuing to travel downstream (for $t<8$ ). The $C 4$ case for $A R=2$ shows some slight upstream motion at the end of the trajectory but is not as district as the lower pitch rate cases. As observed earlier, at $t=8$ the position of the $\mathrm{LEV}$ is in roughly the same position for all pitch rates. For the $A R=4$ plate, the center of the LEV travels the same path for the four pitch rates for roughly one chord length, and then begins to vary. The LEV advects along the same path but with different velocity for different pitch rates as evident from the $x$ - $t$ diagram. The dashed lines in Figure 8 for the $A R=4$ wing represent a second LEV that has developed, which was not observed for the $A R=2$ wing for the shown time frame. The emergence of the second LEV is obstructed due to the initial LEV remaining over the wing surface for a longer period of time. For the 2D plate, the LEV centroid appears to initially take a similar path towards the trailing edge as observed for the $A R=4$ wing (although somewhat higher at $x=1$ ). Once the LEV reaches the trailing edge of the 2D wing, it advects downstream along similar paths for the four pitch rates considered. The difference between the trajectories of the LEV between the $A R=4$ and $2 D$ plates is caused by the lack of tip effects for the two-dimensional wing. The strong tip vortices that develop while the wing pitches induce large downward velocity that pushes the LEV towards the centerline of the wing and restricts it from immediately lifting off. When comparing the two finite aspect ratios, the LEV remains closer to the wing surface for the $A R=2$ plate, which again is due to the tip vortices inducing a relatively stronger downward velocity on the LEV along the midspan plane. For the $A R=4$ plate, the tip vortices concurrently pull fluid into the region between the arch-shaped LEV and the tip vortices that in turn pushes the LEV away from the plate once the LEV reaches a certain size. On the other hand, the tip vortices for the $A R=2$ plate cover a majority of the wing surface, resulting in significant downward induced velocity which restricts any significant upward motion of the LEV.

Evolution of vortex strength (spanwise circulation $\Gamma_{z}$ ) along the midspan is tracked using the second vortex identification method of Graftieaux et al. ${ }^{28}, \gamma_{2}$, to define the boundary of the LEV. Graftieaux et al. ${ }^{28}$ defines regions where $\left|\gamma_{2}\right|>2 / \pi$ to be locally dominated by rotation and therefore represents the core of a vortex. Cutoff values for $\left|\gamma_{2}\right|$ are normally between 0.6 and 0.75 in the literature ${ }^{29,30}$. Since we are interested in the circulation of the LEV, we seek for regions where $\gamma_{2}<-0.75$, and then numerically integrate the spanwise vorticity inside the vortex core boundary to approximate the midspan circulation of the LEV.

Results from the $\Gamma_{z}$ calculations at the midspan plane are given in Figure 9 for the $A R=2,4$, and 2D plates. Each of the solid lines illustrates the growth of the first LEV created by the different pitching motion. The dashed lines represent the development of the second LEV for the $A R=4$ and $2 \mathrm{D}$ plates. For the fast pitching $C 1$ case, the LEV circulation increases almost linearly during the pitching motion for the $A R=2$ plate until it levels off around $t=1.5$. By $t=4$, the circulation increases again and then levels off for the $A R=2$ case. For the $A R=4$ plate, the circulation of the first LEV continues to increase until $t=3$. Afterwards, there is a decrease in its strength. This decrease is due to the detachment of the LEV from the plate (the tail of the first LEV turns into the second LEV) and diffusive vorticity flux escaping through the $\gamma_{2}$ boundary. As can be seen from the dashed lines on the right side of Figure 9, a second LEV begins to develop around this 
time. We observe a similar behavior for the $C 2$ cases for both aspect ratios, but offset by roughly one convective unit.

For the 2D plate, the circulation of the first LEV increases linearly for the $C 1$ case during the pitching interval and then increases again until $t=3$. At this point, the LEV centroid begins to travel away from the surface of the plate, which was observed in Figure 8 . While we expect for the 2D plate that the circulation should monotonically increase until it levels off to a constant value, there is a slight reduction in $\Gamma_{z}$ for the $C 1$ and $C 2$ cases that occurs at the end of the motion (at $t=1$ and 2, respectively). This is due to how the boundary of the $\gamma_{2}$ cutoff contour is generated. While the first LEV is attached to the plate, the $\gamma_{2}$ contour includes part of the shear layer near the leading edge, which provides added strength to the calculated LEV circulation. The observed decease is actually an artifact of added circulation from the high value of vorticity in the vicinity of the leading edge during the pitching motion no longer being captured by the $\gamma_{2}$ contour.

A much slower development of the LEV circulation is observed for all aspect ratios between $t=0$ and 2 for the $C 4$ and $C 6$ pitching cases. The circulation begins to increase at nearly the same rate for the $A R=2$ and 4 plates beyond $t=2$, and then somewhat levels off for the $A R=2$ case around $t=4.5$. We notice for the $A R=4$ plate, the calculated circulation begins to decrease after it reaches its maximum value around $t=5$ due to the pinch off of the first LEV. The tail of the LEV structure is no longer included in the first $\gamma_{2}$ contour which results in the apparent decrease in $\Gamma_{z}$. The second LEV forms with the excluded vorticity and leads to the growth of the second LEV, shown in Figure 9. For both the $A R=4$ and $2 \mathrm{D}$ plates, the midspan circulation of the second LEV begins to increase at a similar time for the four pitch rates, but the rate at which it increases is greater for the $2 \mathrm{D}$ plate.

$A R=2$

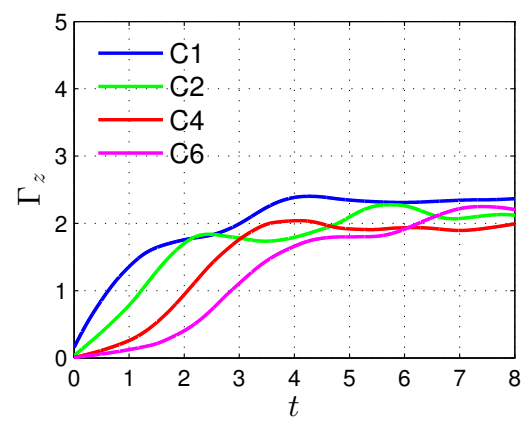

$A R=4$

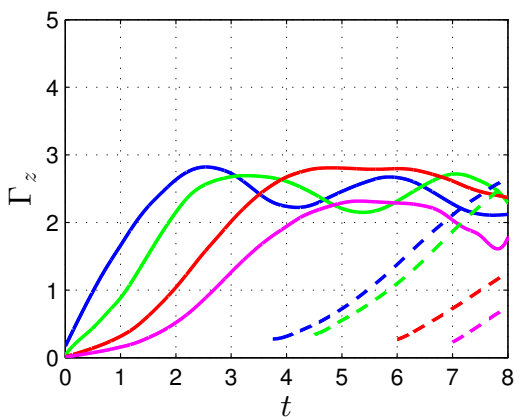

$2 D$

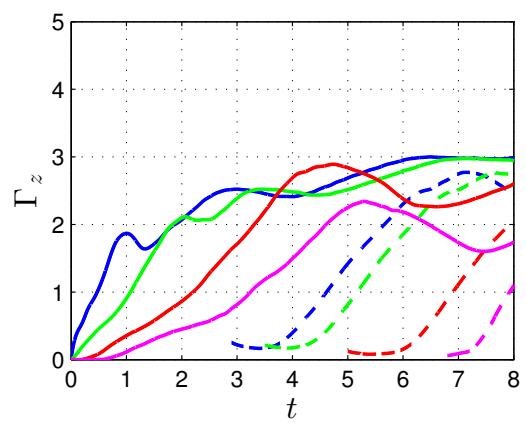

FIG. 9: Comparison of the temporal evolution of the midspan circulation for the four pitch rates due to the LEV for the $A R=2$ (left), $A R=4$ (center), and 2D (right) flat plate.

The main difference between the flows around $A R=2$ and 4 plates is how the LEV circulation grows. For plate of $A R=2$, the LEV is influenced significantly by tip effects, keeping the roll up of the attenuating streamwise convection and detachment of the LEV to take place directly above the wing. For the $A R=4$ plate, the reduced tip effect allows for the LEV to lift up and deform more freely, as shown by Figures 6 and 7. This in turn allows for the increased circulation to be accumulated by the LEV. The first LEV grows large to critical threshold, allowing for the second LEV to start forming earlier than the case with $A R=2$. 


\section{AERODYNAMIC FORCES}

Figure 10 shows the temporal evolution of the aerodynamic lift and drag coefficients for the $A R=2,4$, and 2D plates undergoing the four pitching motions considered in this study. For all pitch rates and aspect ratios considered, there is a spike in $C_{L}$ centered around $t=0$ due to non-circulatory ${ }^{6}$ effects from the angular acceleration of the wing. The amplitude of the peak is related to the smoothing value $a$ in Eq. (1), where larger values of a result in greater peak values. As the plate continues to pitch, $C_{L}$ begins to increase to a second maximum value for all pitch rates considered. This second increase in $C_{L}$ is attributed to suction from the LEV.

As the pitch rate is decreased, we notice a substantial reduction in the slope of the lift curve between the first three pitch rates $(C 1, C 2$, and $C 4)$, but a more gradual reduction between the two slowest cases, $C 4$ and $C 6$. By the end of the pitching motion, there is a sharp reduction in lift due to the angular deceleration. After the plate has completed its motion, the $C_{L}$ curves for $A R=2$ collapse. For $A R=4$, the $C_{L}$ curves for the $C 1$ and $C 2$ cases collapse $t \geq 4$ and then gradually begin to increase again after $t=5$ due to the development of a second LEV. We also observe a similar collapse between the $C 4$ and $C 6$ curves by $t=6.5$, although there is no gradual secondary increase in lift due to further LEV development.

$A R=2$
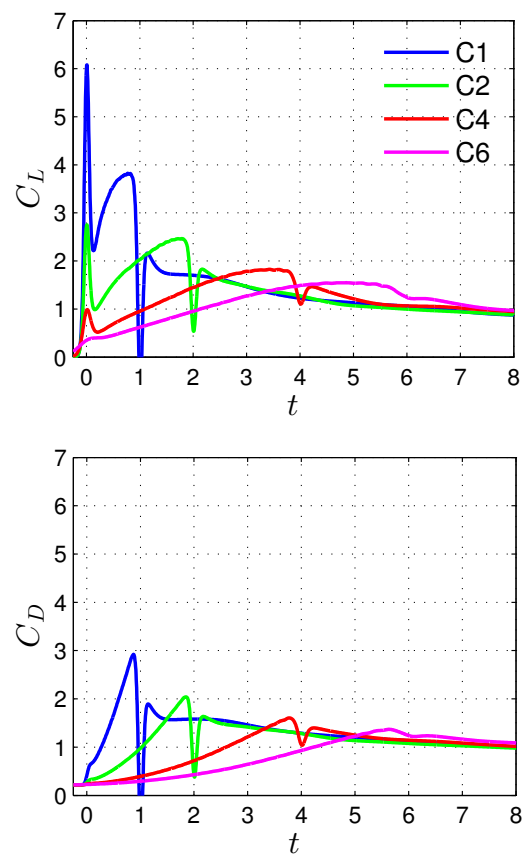

$A R=4$
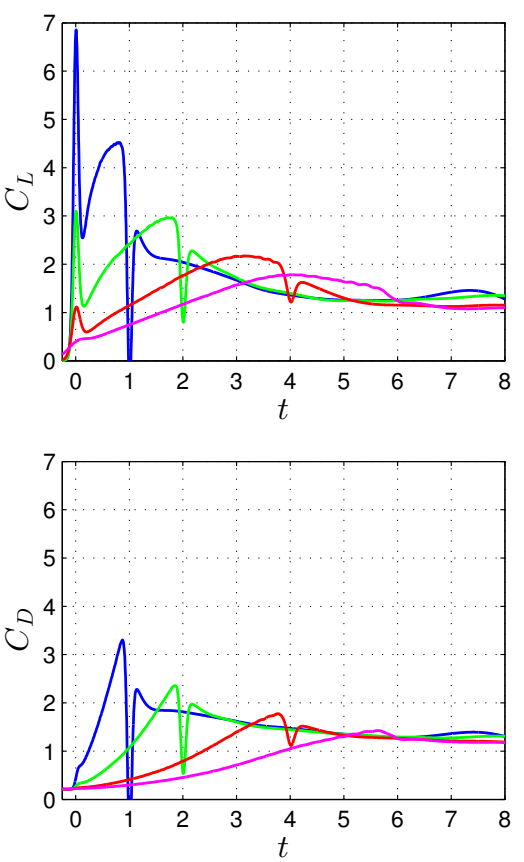

$2 \mathrm{D}$
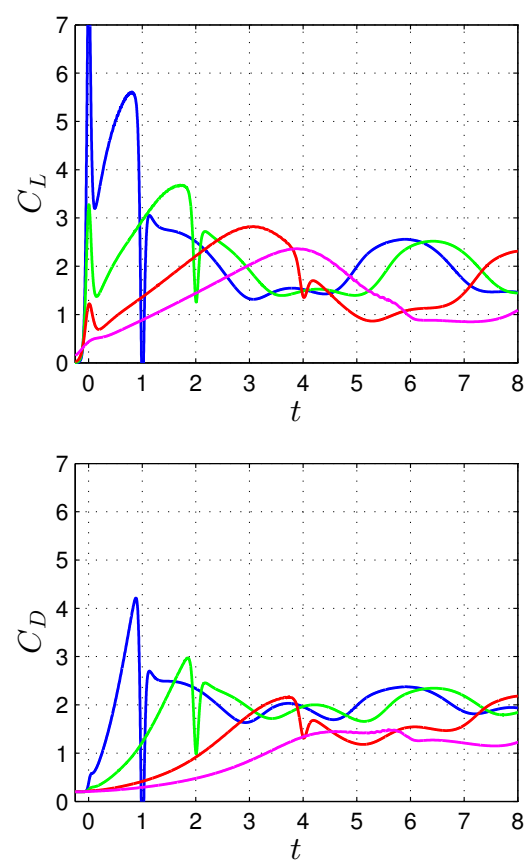

FIG. 10: Aerodynamic force coefficients for various pitch rates at $R e=300$ for $A R=2$, $A R=4$, and $2 \mathrm{D}$ plates.

An interesting observation made between the three aspect ratios is the time at which the maximum $C_{L}$ is attained for the $C 4$ and $C 6$ cases. For the $A R=2$ plate, the maximum $C_{L}$ is attained at $t=3.5$ and $t=5$ for the $C 4$ and $C 6$ cases, respectively. For both the $A R=4$ and $2 \mathrm{D}$ plates, the maximum lift is achieved at about half a convective time earlier for $C 4$ case (at $t=3$ ) and nearly two convective units for the $C 6$ case (at $t=4$ ). After 
this maximum is achieved, there is a decrease in $C_{L}$ for both the $A R=4$ and $2 \mathrm{D}$ plates, signifying that the LEV lifts off earlier in time when compared to the $A R=2$ plate for the slower pitching rates. We note that the maximum lift being achieved before the end of the pitching motion is due to the formation and detachment of the LEV, which provides the enhanced lift. This lift off of the LEV for the case of $A R=4$ is evident in Figures 7 and 8. In comparison, we have observed that the LEV for the case of $A R=2$ stays relatively close to the plate throughout the time shown in the figures.

The second row of Figure 10 presents the $C_{D}$ histories for the $A R=2,4$, and $2 \mathrm{D}$ plates. The initial drag value is the steady-state value at zero incidence. As the pitch ramp begins, drag increases due to the increase in the projected area seen by the flow. At the end of the motion, there is a negative spike in $C_{D}$ due to the deceleration of the motion. For the $C 1$ cases for the three aspect ratios, this rapid deceleration actually results in a slight thrust. The $C_{D}$ curves for all pitch rates for the $A R=2$ plate collapse to one another after the respective pitching motion is complete. This is also observed for the $A R=4$ plate except for the $C 1$ and $C 2$ pitching cases after $t=6$ due to the slight increase in drag resulting from the development of the second LEV. For the $2 \mathrm{D}$ wing, there is no collapse in the $C_{L}$ or $C_{D}$ curves due to the subsequent development of additional LEV's.

$A R=2$
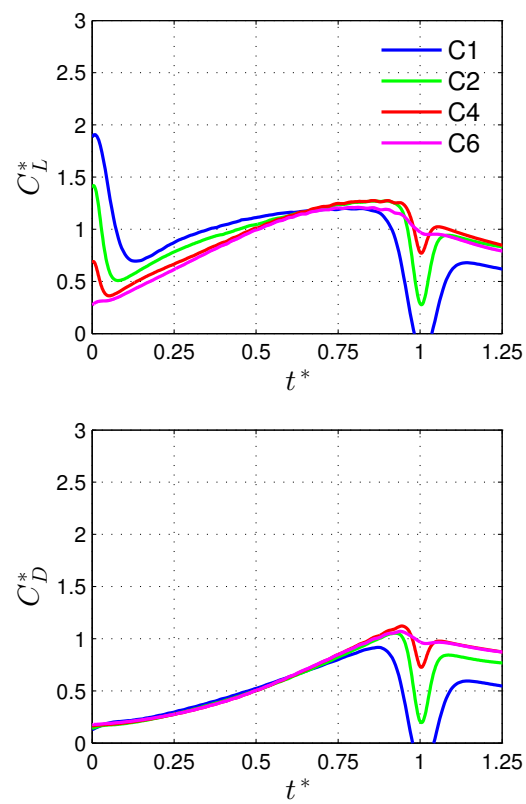

$A R=4$
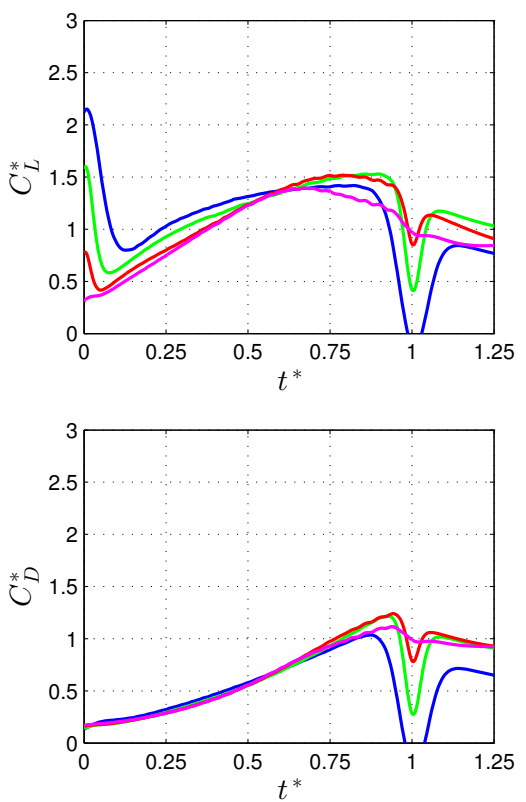

$2 \mathrm{D}$
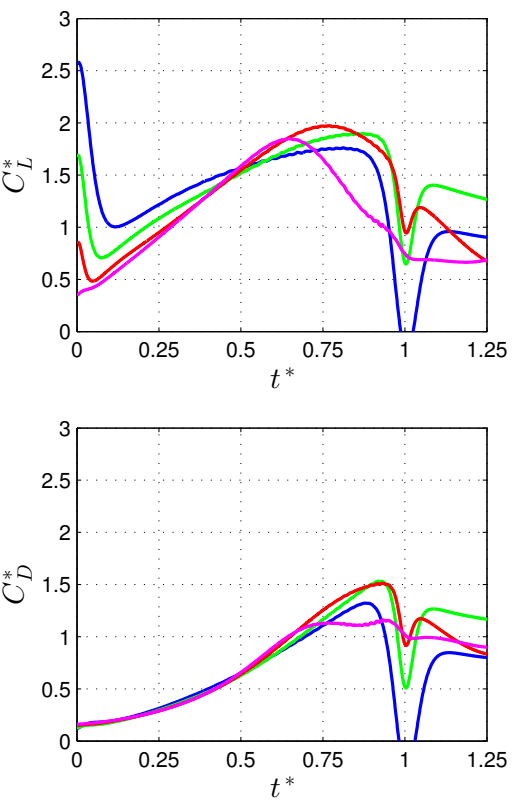

FIG. 11: Scaled lift and drag coefficients over scaled time for various pitch rates at $R e=300$ for the $A R=2, A R=4$, and 2D plates.

Parameter study of various acceleration rates suggests opportunity for non-dimensional scaling. During the pitching motion, the total streamwise travel distance and therefore velocity varies along chord of the plate. At the leading edge, where the pivot point is located, the plate travels at a speed of $U_{\infty}$. As the plate is pitching, the velocity of the plate increases along the chord of the wing from the minimum value of $U_{\infty}$ at the pivot point to $\max _{t}\left(\left\|\mathbf{U}_{\infty}\right\|+\left\|\mathbf{u}_{\mathrm{TE}}\right\|\right)$ at the trailing edge. For the present scaling analysis, we include the trailing-edge speed into the characteristic velocity used in the non-dimensionalization of the 
lift and drag coefficients. The scaled lift and drag coefficients are given by

$$
C_{L}^{*}=\frac{F_{x}}{\frac{1}{2} \rho U_{\text {char }}^{2} A} \quad C_{D}^{*}=\frac{F_{y}}{\frac{1}{2} \rho U_{\text {char }}^{2} A},
$$

where $U_{\text {char }}=U_{\infty}+u_{\mathrm{TE}, \max }$, and $u_{\mathrm{TE}, \max }=\max _{t}\left\|\mathbf{u}_{\mathrm{TE}}\right\|=c \dot{\alpha}=2 c K$ is the maximum velocity of the trailing edge during the pitching motion. The maximum velocity achieved by the trailing edge occurs during the constant angular velocity $(\dot{\alpha})$ portion of the pitching motion. We also scale the time variable with $t^{*}=t / t_{p}$, where $t_{p}=\alpha_{\max } / 2 \mathrm{~K}$ is the pitching interval previously defined for Eq. (1). Figure 11 presents the scaled lift (top) and drag (bottom) coefficients $\left(C_{L}^{*}\right.$ and $C_{D}^{*}$, respectively) over the scaled convective time, $t^{*}$. Here, we observe a good collapse in both scaled lift and drag curves for all pitch rates and aspect ratios considered. This collapse of data confirms that reduced frequency $K$ is the driving variable that determines the unsteady force. The definition of the characteristic velocity used above has resemblance to how Milano and Gharib ${ }^{31}$ chose the characteristic leadingedge velocity for flapping plates. We have also considered the use of $\max _{t}\left\|\mathbf{U}_{\infty}+\mathbf{u}_{\mathrm{TE}}\right\|$ as the characteristic velocity for the non-dimensionalization of the forces. We found that this choice of characteristic velocity does not collapse the force histories well for the various acceleration rates. This suggest that the generation of the hydrodynamic forces on the plate is due to the additive effects from the freestream interacting with the leading edge and the trailing edge undergoing a rotational motion. Therefore, we use the previously defined characteristic value based on the sum of the norms, $U_{\infty}+u_{\mathrm{TE} \text {,max }}$.

Alternatively, the forces can be collapsed by considering the lift-to-drag ratio $(L / D)$ since such scaling does not require the use of characteristic velocity in the definition. For each aspect ratio, we present $L / D$ in Figure 12 with the time variable scaled in the same manner, $t^{*}=t / t_{p}$. At the beginning of the motion, we observe large peaks in the $L / D$ curves due to the initial angular acceleration. After that initial peak, we observe a collapse in the $L / D$ curves for the four acceleration rates. For the three aspect ratios considered, the collapse of the $L / D$ curves is qualitatively similar. As the plate increases its angle of attack from $\alpha=0^{\circ}$ to $45^{\circ}$, the $L / D$ curves decrease resulting from the increased pressure drag due to the highly separated wake.
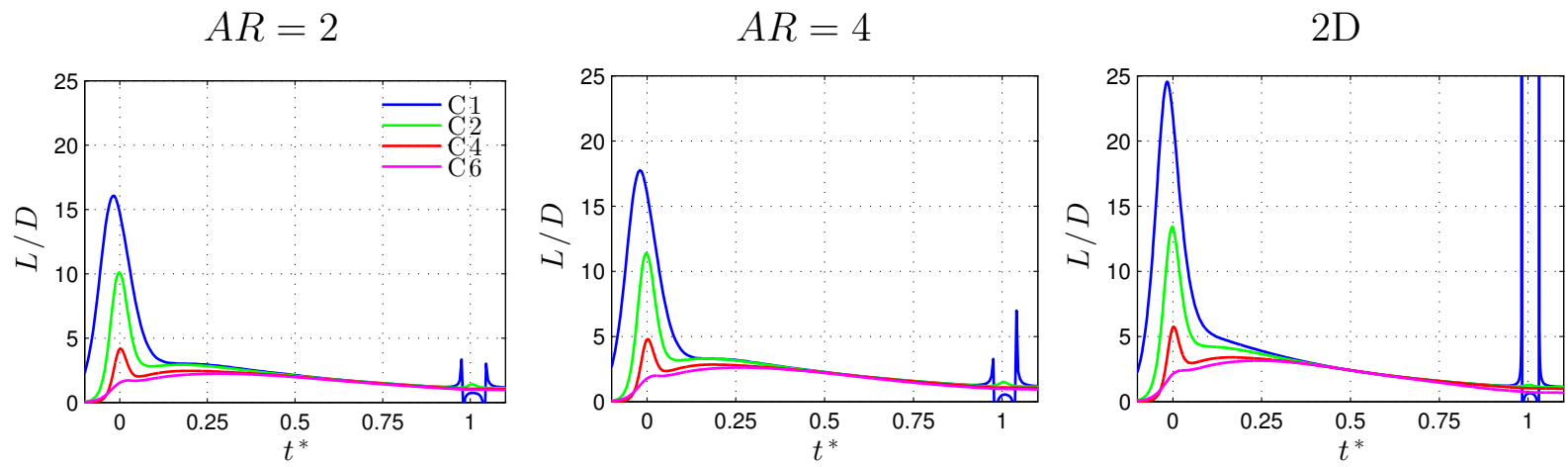

FIG. 12: Lift-to-drag ratios over scaled time for various pitch rates at $R e=300$ for the $A R=2, A R=4$, and $2 \mathrm{D}$ plates. 
$A R=2$
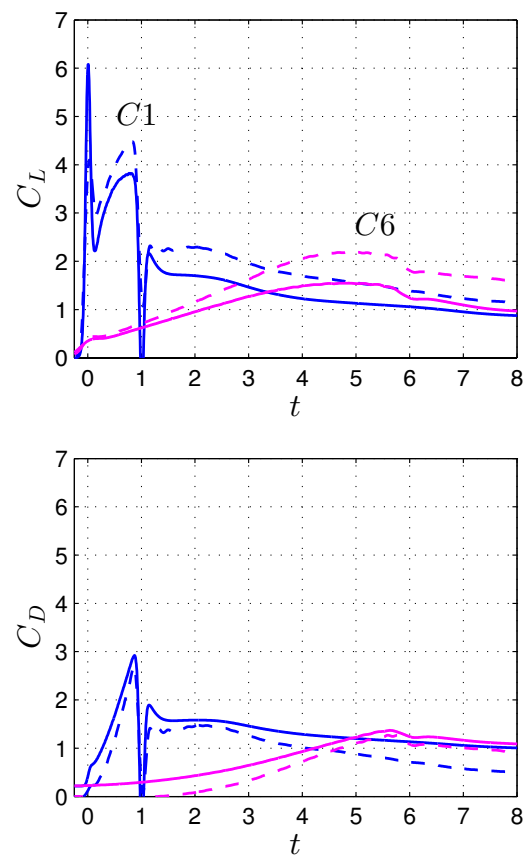

$A R=4$
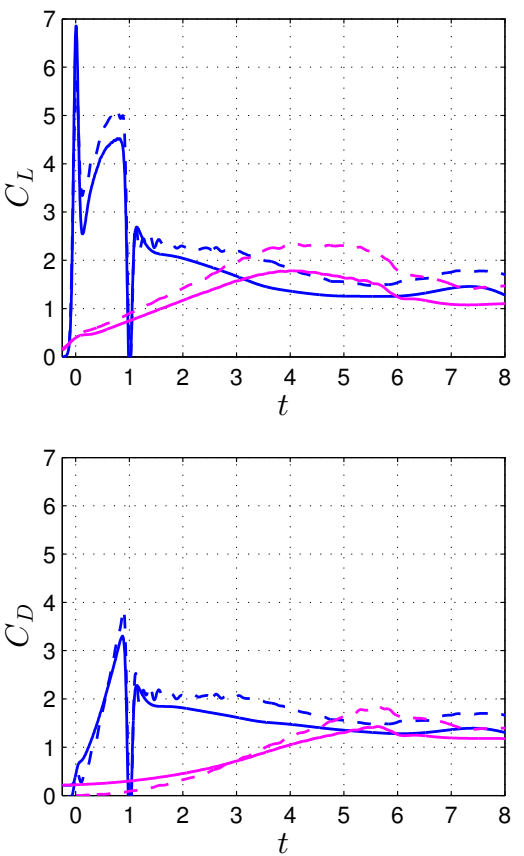

$2 \mathrm{D}$
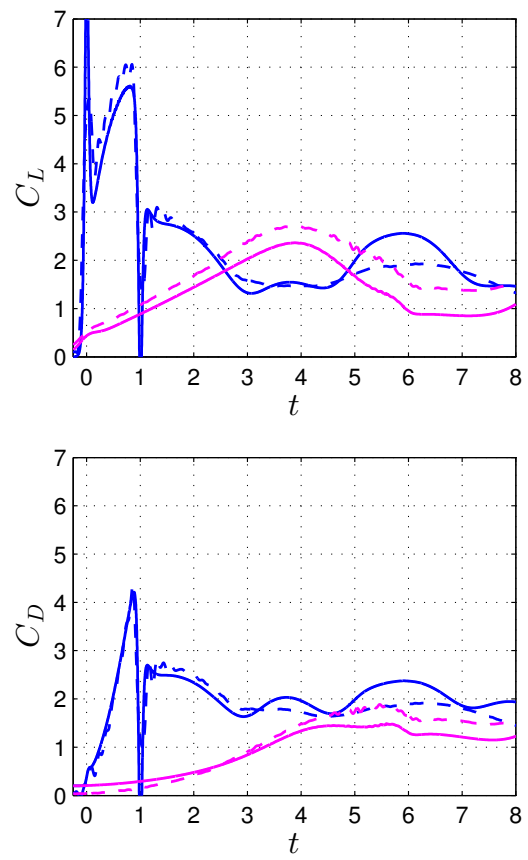

FIG. 13: Comparison between the experimental $(R e=20,000$, dashed $)$ and present simulation $(R e=300$, solid) force coefficients for the $C 1$ and $C 6$ pitch rates with $A R=2$, 4 , and $2 \mathrm{D}$ plates.

\section{EFFECTS OF REYNOLDS NUMBER}

We examine the aerodynamic force coefficients for the $A R=2,4$, and 2D pitching plates from the simulation at $R e=300$ and the experiments at $R e=20,000$ in Figure 13. The first row compares the $C_{L}$ curves for $A R=2, A R=4$, and 2D plates pitching from 0 to $45^{\circ}$ about the leading edge for the $C 1$ and $C 6$ cases. We note that the $2 \mathrm{D}$ simulations are performed in only in the $x y$-plane, whereas the $2 \mathrm{D}$ experiments are conducted with a flat plate that extends wall to wall within the water tunnel $(\approx 1 \mathrm{~mm}$ tip gap $)$. For the $C 1$ cases, a large non-circulatory spike in lift is present centered around $t=0$ resulting from the fast acceleration of the plate. This non-circulatory spike is less evident for the $C 6$ cases due to the reduced $a$ value which smooths the motion profile (Eq. (1)), and due to lower acceleration at lower pitch rate.

As the plate continues to increase its angle of attack, $C_{L}$ increases due to the development of a leading-edge (dynamic stall) vortex over the plate surface. After the pitching motion is complete $\left(\alpha=\alpha_{\max }=45^{\circ}\right.$ has been attained), we observe a reduction in $C_{L}$ over time for all plates. It is interesting to note the qualitative comparison between the long-term force histories for the $A R=4 C 1$ case, where an increase in CL is observed for experimental and computational results around $t=7$ due to the development of a second LEV. We observe similar comparisons between the $C_{D}$ curves, with the main difference being the initial drag value before the plate begins to pitch, which is expected due to increased viscous effects at $R e=300$. Note that the lift-to-drag ratio is close to unity, signifying that the resultant aerodynamic force is primarily normal to the plates surface. Although there are two orders of magnitude of difference in Reynolds number between simulations and experiments, we 
observe reasonable agreement in the forces for all aspect ratios considered. Differences in the aerodynamic forces between the two results should be expected due to the noticeable viscous losses present in the low Reynolds number simulations. The enhanced diffusion of vorticity can account for the reduced lift slopes and maximum force values. While the magnitude of the force coefficients deviate somewhat between the two, we observe similar behavior of when maxima in $C_{L}$ and $C_{D}$ are achieved, and also similar characteristics even after the pitching motion ends.

Figure 14 presents a comparison between the flow field around the $A R=4$ plate for the $C 1$ case. The experimental fluorescent dye flow visualization (left column) was obtained along the $3 / 4,7 / 8$, and tip spanwise locations and is compared to the magnitude of vorticity from the simulation at the same locations (right column). The vorticity magnitude contours $(3 \leq\|\boldsymbol{\omega}\| \leq 15)$ were chosen to match the experimental dye injection photos. The results are presented in a plate-fixed reference frame at $\alpha=23^{\circ}, 32^{\circ}$, and $45^{\circ}$ corresponding to $t=0.5,0.75$, and 1 , respectively. As the angle of attack reaches $23^{\circ}$, we observe similar features between the experiments and computations, namely the shedding of the starting vortex from the trailing-edge, the development of the tip vortices, and the initial roll up of the vortex sheet from the leading edge forming the LEV. The obvious difference between the two flow fields is the absence of the Kelvin-Helmholtz instability in the shear layer at the trailing edge at the Reynolds number of the computation.

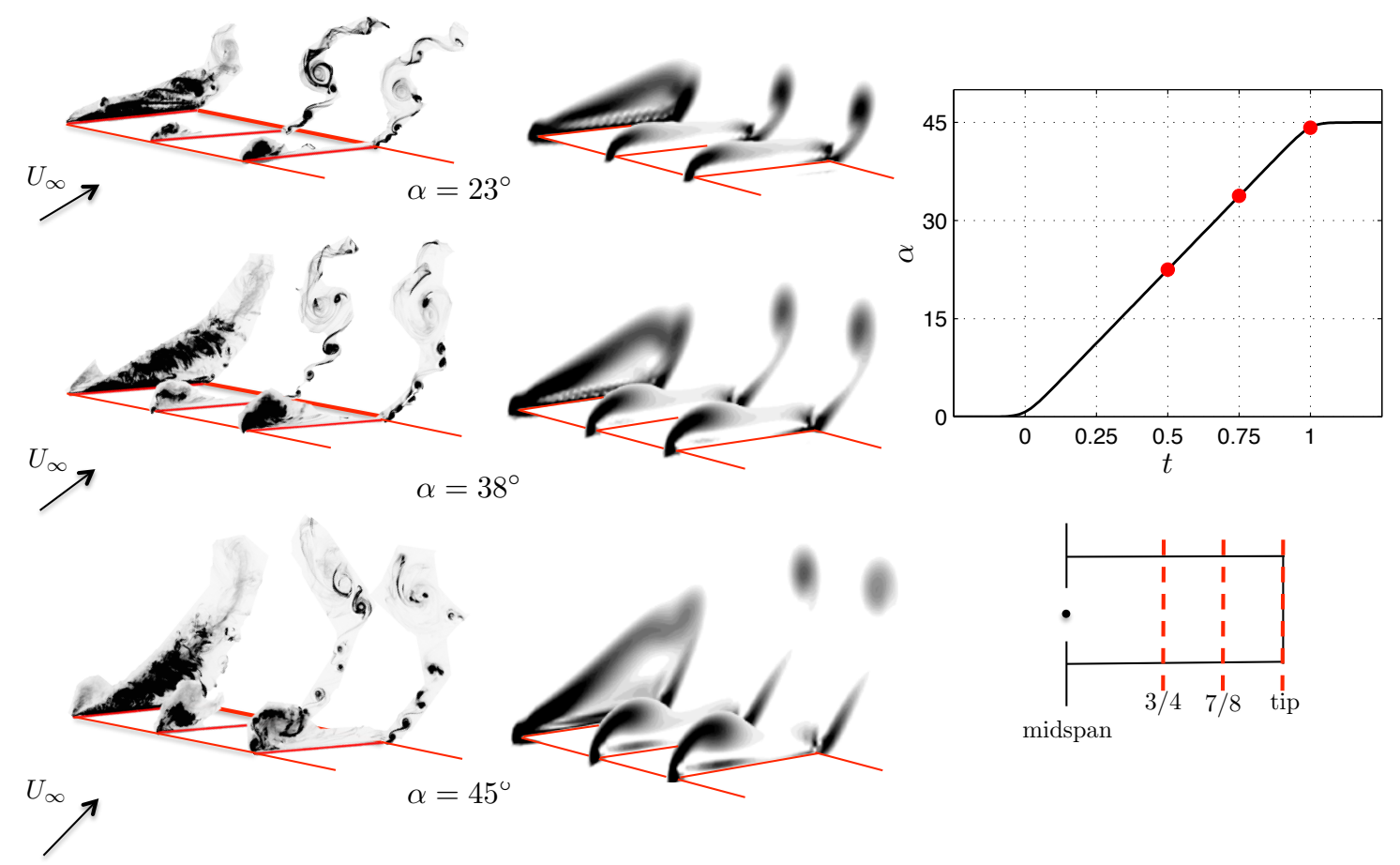

FIG. 14: Comparison between experimental fluorescent dye flow visualizations at $R e=20,000$ (left) and computed contours of vorticity magnitude $(3 \leq\|\boldsymbol{\omega}\| \leq 15)$ slices from the present simulations at $R e=300$ (right), taken along the $3 / 4,7 / 8$, and tip span

locations for the $A R=4$ plate pitching from 0 to $45^{\circ}$ over one chord of travel $(C 1)$.

Clear differences between the development of the LEV between the experiment and computation are observed as the plate continues to increase its angle of attack. The streamwise 
elongation of the LEV core for the low Reynolds number is observed for DNS, whereas for the LEV is more compact towards the leading edge of the plate for higher Reynolds number experiment. We believe this to be the reason between the difference in the $C_{L}$ curves given in Figure 13, since the compact LEV has a stronger suction associated with it compared to the elongated LEV, which creates a more profound suction on the top surface of the wing, thus leading to a higher $C_{L}$. Although the differences in Reynolds number between the two results is of two orders of magnitude and there is a lack of any instabilities in the present numerical results, there is a good agreement in the qualitative behavior of the wake vortices which leads us to believe that the present simulations are valid for the vortex dynamics around low-aspect-ratio pitching plates, especially in the developmental stage of vortex formation.

\section{CONCLUDING REMARKS}

Three-dimensional direct numerical simulations via the immersed boundary projection method have been performed to examine the vortex dynamics for pitching flat plates of aspect ratio 2, 4 and 2D, and compared with experiment in a water tunnel, at a range of pitch rates that span across the vortex formation time. While the Reynolds number is 300 in the computation and 20,000 in the experiments, the results were in good agreement in terms of the wake structures and lift and drag histories. The differences between the two studies were the appearance of Kelvin-Helmholtz instabilities and the compactness observed for the LEV core found in the experiments, which are both attributed to Reynolds number effects.

During the early phase of pitching, the tip vortices do not affect the flow field near the midspan significantly. However, as the tip vortices grow, tip effects influence the wake dynamics in a global manner. While the $A R=2$ plate kept the LEV to remain in the vicinity of the wing, the reduced tip effects from the $A R=4$ plate allowed the LEV to detach earlier and led to the subsequent formation of the second LEV. The first LEV detachment caused the tip vortices to deform and interact strongly with the LEV structure that resembles the arch-type vortex. The detaching LEV creates a peak in the lift force before the end of motion for the slower pitching cases. For both the $A R=2$ and 4 plates, lift and drag histories for the examined pitch rates evinced good collapse to a common curve, if coefficients are normalized by the running speed of the trailing edge, instead of the free-stream relative speed.

\section{ACKNOWLEDGMENTS}

RJ and KT were supported by the 2012 USAF Air Vehicles Directorate Summer Research and Development Program and the 2013 ASEE Summer Faculty Fellowship Program during their stays at the Wright-Patterson Air Force Base. RJ also acknowledges the AeroPropulsion, Mechatronics, and Energy Fellowship from the Florida State University.

\section{REFERENCES}

${ }^{1}$ T. J. Mueller, ed., Fixed and flapping wing aerodynamics for micro air vehicle applications (AIAA, 2001).

${ }^{2}$ D. J. Pines and F. Bohorquez, "Challenges facing future micro-air-vehicle development," J. Aircraft 43, 290-305 (2006). 
${ }^{3}$ S. Watkins, J. Milbank, B. J. Loxton, and W. H. Melbourne, "Atmospheric winds and their implications for microair vehicles," AIAA J. 44, 2591-2600 (2006).

${ }^{4}$ K. Granlund, M. Ol, B. Monnier, and D. Williams, "Airfoil longitudinal gust response in separated vs. attached flows," (42nd AIAA Fluid Dynamics Conference and Exhibit (AIAA2012-2695), 2012).

${ }^{5}$ G. Leishman, Principles of helicopter aerodynamics, 2nd ed. (Cambridge Univ. Press, 2006).

${ }^{6}$ T. Theodorsen, "General theory of aerodynamic instability and the mechanism of flutter," Tech. Rep. 496 (NACA, 1935).

${ }^{7} \mathrm{~K}$. Taira and T. Colonius, "Three-dimensional flows around low-aspect-ratio flat-plate wings at low Reynolds numbers," J. Fluid Mech. 623, 187-207 (2009).

${ }^{8}$ J. H. J. Buchholz and A. J. Smits, "On the evolution of the wake structure produced by a low aspect ratio pitching panel," J. Fluid Mech. 546, 433-443 (2006).

${ }^{9}$ M. A. Green, C. W. Rowley, and A. J. Smits, "The unsteady three-dimensional wake produced by a trapezoidal panel." J. Fluid Mech. 685, 117-145 (2011).

${ }^{10}$ D. J. Garmann, M. R. Visbal, and P. D. Orkwis, "Three-dimensional flow structure and aerodynamic loading on a revolving wing." Phys. Fluids 25, 034101-27 (2013).

${ }^{11} \mathrm{~A}$. Jones and H. Babinsky, "Unsteady lift generation on rotating wings at low Reynolds numbers." J. Aircaft 47, 1013-1021 (2010).

${ }^{12} \mathrm{~K}$. K. Chen, T. Colonius, and K. Taira, "The leading-edge vortex and quasisteady vortex shedding on an accelerating plate," Phys. Fluids 22, 033601-11 (2010).

${ }^{13}$ C. W. P. Ford, R. Stevens, and H. Babinsky, "Flexible leading edge flap on an impulsively started flat plate at low Reynolds numbers." (50th AIAA Aerospace Sciences Meeting (AIAA2012-2840), 2012).

${ }^{14}$ R. Stevens, C. W. P. Ford, and H. Babinsky, "Experimental studies of an accelerating, pitching, flat plate at low Reynolds numbers." (51st AIAA Aerospace Sciences Meeting (2013-0677), 2013).

${ }^{15}$ K. O. Granlund, M. V. Ol, and L. P. Bernal, "Unsteady pitching flat plates," J. Fluid Mech. 733, R5.1-13 (2013).

${ }^{16} \mathrm{H}$. Yu and L. P. Bernal, "Effect of pivot point on aerodynamic force and vortical structure of pitching flat plate wings," (51st AIAA Aerospace Sciences Meeting (AIAA 2013-0792), 2013).

${ }^{17}$ K. Granlund, M. Ol, K. Taira, and R. Jantzen, "Parameter studies on rotational and translational accelerations of flat plates," (51st AIAA Aerospace Sciences Meeting (AIAA20130068), 2013).

${ }^{18}$ M. R. Visbal, "Flow structure and unsteady loading over a pitching and perching lowaspect-ratio wing," (42nd AIAA Fluid Dynamics Conference and Exhibit (AIAA 20123279), 2012).

${ }^{19}$ D. J. Garmann and M. R. Visbal, "Numerical investigation of transitional flow over a rapidly pitching plate," Phys. Fluids 23, 094106 (2011).

${ }^{20}$ R. Jantzen, K. Taira, K. Granlund, and M. Ol, "On the influence of pitching and acceleration on vortex dynamics around a low-aspect-ratio rectangular wing," (51st AIAA Aerospace Sciences Meeting (AIAA2013-0833), 2013).

${ }^{21}$ M. Gharib, E. Rambod, and K. Shariff, "A universal time scale for vortex ring formation," J. Fluid Mech. 360, 121-140 (1998).

${ }^{22}$ A. C. DeVoria and M. J. Ringuette, "Vortex formation and saturation for low-aspect-ratio rotating flat-plate fins," Exp. Fluids 52, 441-462 (2012). 
${ }^{23}$ J. D. Eldredge, C. Wang, and M. V. Ol, "A computational study of a canonical pitch-up, pitch-down wing maneuver," (39th AIAA Fluid Dynamics Conference (AIAA2009-3687), 2009).

${ }^{24}$ T. O. Yilmaz and D. Rockwell, "Flow structure on finite-span wings due to pitch-up motion," J. Fluid Mech. , 518-545 (2012).

${ }^{25} \mathrm{~K}$. Taira and T. Colonius, "The immersed boundary method: a projection approach," J. Comput. Phys. 225, 2118-2137 (2007).

${ }^{26} \mathrm{~T}$. Colonius and K. Taira, "A fast immersed boundary method using a nullspace approach and multi-domain far-field boundary conditions," Comput. Methods Appl. Mech. Engrg. 197, 2131-2146 (2008).

${ }^{27}$ M. V. Ol, L. Bernal, C.-K. Kang, and W. Shyy, "Shallow and deep stall for flapping low Reynolds number airfoils," Exp. Fluids 46, 883-901 (2009).

${ }^{28}$ L. Graftieaux, M. Michard, and N. Grosjean, "Combining PIV, POD and vortex identification algorithms for the study of unsteady turbulent swirling flows," Meas. Sci. Tech. 12, 1422-1429 (2001).

${ }^{29}$ A. R. Jones and H. Babinsky, "Reynolds number effects on leading edge vortex development on a waving wing," Exp. Fluids 51, 197-210 (2011).

${ }^{30}$ Y. S. Baik, L. P. Bernal, K. Granlund, and M. V. Ol, "Unsteady force generation and vortex dynamics of pitching and plunging aerofoils," J. Fluid Mech. 709, 37-68 (2012).

${ }^{31}$ M. Milano and M. Gharib, "Uncovering the physics of flapping flat plates with artificial evolution," J. Fluid Mech. 534, 403-409 (2005). 